\title{
Review of Algorithms for Improving Control of Blood Glucose Levels
}

\author{
Jelena Tašić, György Eigner, and Levente Kovács \\ Physiological Controls Research Center \\ Óbuda University \\ Budapest, Hungary \\ Email: tasic.jelena@phd.uni-obuda.hu, eigner.gyorgy@nik.uni-obuda.hu,kovacs@uni-obuda.hu
}

\begin{abstract}
In this study, we review recently proposed advanced methods based on various techniques in order to improve blood glucose control while avoiding an increased risk of hypoglycemia or hyperglycemia in patients with type 1 diabetes. We introduce type 1 diabetes and the artificial pancreas and provide an overview of research advances based on predictive control, statistical processes, filters, and machine learning. Common control methods have been successfully used to control blood glucose levels while new approaches that rely on machine learning algorithms offer promising performance.
\end{abstract}

Index Terms-Type 1 diabetes, artificial pancreas, continuous glucose monitoring, model predictive control, PID control, Kalman filter, neural networks, machine learning

\section{INTRODUCTION}

Type 1 diabetes mellitus (T1DM) is an autoimmune disease that destroys pancreatic $\beta$-cells responsible for production of the insulin peptide hormone that enables maintaining blood glucose (BG) levels. Patients with T1DM require exogenous insulin administration due to the lack of internal insulin production. However, external administration has its own risks. For example, physical exercise and applied insulin therapy may lead to dangerous hypoglycemia. In general, caution should be taken when using insulin as it may cause hypoglycemia (BG $<70 \mathrm{mg} / \mathrm{dL}$ ) leading to a potential seizure, coma, or even death of a patient. Underdosing may also cause persistent hyperglycemia (BG $>180 \mathrm{mg} / \mathrm{dL}$ ) leading to long term complications such as neuropathies, nephropathy, or cardiovascular diseases [1].

In its simplest form, the artificial pancreas (AP) is a closedloop glucose controller consisting of a wearable insulin pump that delivers insulin based on Continuous Glucose Monitoring (CGM) system that measures the BG levels. A continuous subcutaneous insulin infusion (CSII) pump communicates with the CGM system that measures the BG levels [2]. After calculating the amount of insulin, the pump releases and delivers the appropriate dose into the patient's body using a control algorithm.

Research results have shown that compared to conventional insulin therapy (open-loop control), AP may control the BG

This project has received funding from the European Research Council (ERC) under the European Union's Horizon 2020 Research and Innovation Programme (grant agreement No. 679681). Project No. 2019-1.3.1-KK-201900007 has been implemented with the support provided from the National Research, Development and Innovation Fund of Hungary, financed under the 2019-1.3.1-KK funding scheme. levels better than the conservative insulin administration and reduce the risk of hypoglycemia. However, numerous challenges in designing AP algorithms need to be considered. The metabolic glucose process may be disturbed under various factors such as dietary changes, unannounced physical exercise, alcohol consumption, circadian rhythm, chronic metabolic variations, menstrual cycle, or insulin sensitivity [3]. Furthermore, security issues, urgent time requirements, and unknown analytical relationship between adjusted parameters and measured values are some of the factors that present additional challenges for developing AP algorithms [4].

After introducing T1D and AP, we provide overview, comparison, and results of recently proposed approaches based on control methods, sensitivity analysis, filters, and machine learning. In Section II, we review control methods based on model predictive control, Bayesian optimization, sliding mode control, proportional integral derivative control, active disturbance rejection control, and bioinspired AP. A short review of a method for identifiability of parameters was given in Section III while methods based on filters are described in Section IV. Novel approaches based on machine learning are reviewed in Section V. We conclude with Section VI.

\section{Approaches Based on Control Methods}

In this Section, we review recently proposed methods for controlling BG levels and reducing the delay of insulin absorption. The model predictive control is one of the most commonly used AP methods [5]. It is used to determine BG levels and optimize insulin delivery through the prediction horizon. Appropriate control may handle meal announcements, limit insulin infusion rates, and BG levels in a simple and proactive manner [6]. Other approaches include Bayesian optimization, sliding mode control, proportional-integral-derivative, active disturbance control, and bio inspired AP. Proportional-integral-derivative is a common approach and its model-based approach relies on physiological models that consider the operation of metabolism.

\section{A. Model Predictive Control Approach}

Algorithms based on the linear model predictive control (MPC) method have been tested in clinical studies and have shown to be able to stabilize BG levels [7]-[9]. They also improve the bolus calculator to more efficiently manage meals. 
Current calculators assume a linear relationship between the size of the announced meal and the insulin bolus and, hence, the correction depends on the BG levels and the insulin intakes [10].

Cairoli et al. [2] improved the MPC method using Signal Temporal Logic (STL) to solve the BG control problem. The Hovorka compartment ordinary differential equation (ODE) model was used for the MPC implementation as shown in Fig. 1. The simulation was performed using the MPC and MPC-STL controllers. The results indicated that STL allowed soft constraints and provided safe BG trajectories without restrictions even at higher meal disturbances. Hence, it enabled avoiding both hypoglycemia and prolonged hyperglycemia.

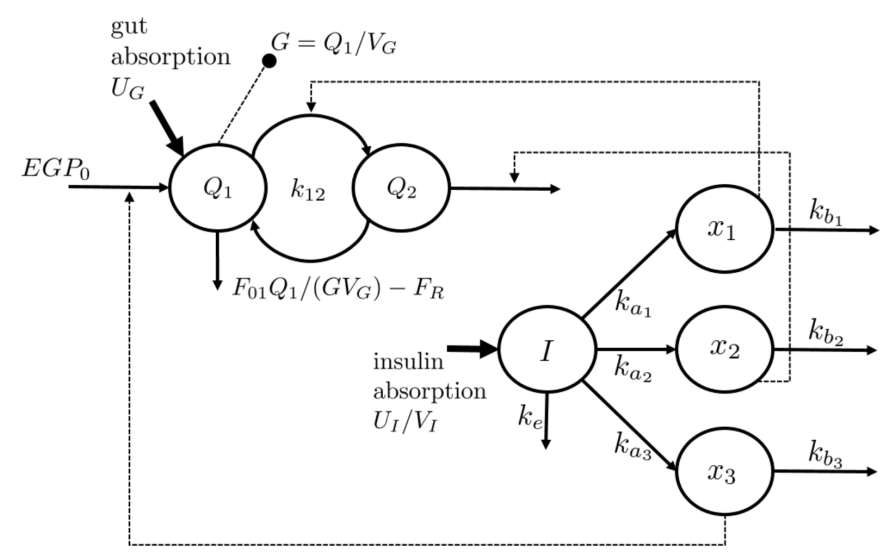

Fig. 1. Hovorka compartment ordinary differential equation model [2].

Shi et al. [11] proposed a zone MPC (ZMPC) method using a dynamic cost function to update the real-time control penalty parameters to reduce the BG levels. The proposed method offered good performance in case of announced and moderate meal-bolus and unannounced meals and exercises. It improved BG levels and rate of insulin delivery to be within a safe range and without the risks of hypoglycemia.

The control algorithm based on the nonlinear MPC (NMPC) method for solving optimal regulation problem (OCP) was presented by Boiroux and Jørgensen [6]. The insulin injection rate was separated into a basal insulin infusion and the administered insulin bolus by using the objective function. The basal insulin was used for endogenous BG production while the bolus administration mitigated the absorption of the carbohydrates (CHO). The improved bolus calculator based on the proposed optimal control algorithm may optimize insulin levels and suspension time relative to a meal size. The algorithm based on the NMPC method enables to more systematically handle the physiological model of a patient. However, the method has disadvantages in designing numerical optimization routines and recognizing physiological models.

Chakrabarty et al. [12] proposed implementation of the embedded ZMPC method using the Fast Adaptive Memetic Algorithm (FAMA) and fast Alternating Direction Method of Multipliers (FADMM) algorithm to solve convex constraints of the linear MPC method. The generated closed-loop data were used to select the optimization algorithm and the appropriate setting parameters. A comparison between the median rate of insulin delivery in euglycemia (announced meals) and equivalent yielding test (unannounced meals) indicated that the proposed method was compatible with other embedded systems and may maintain regulation of the BG levels.

Rashid et al. [13] proposed a novel adaptive MPC method for measuring the optimal insulin infusion rate for considered bounds of the plasma insulin concentration (PIC) to prevent delays in the insulin action. The controller was designed to regulate the BG levels in the presence of significant disturbances (unannounced meals). The recursive subspace-based empirical modeling algorithm based on the predictor-based subspace identification (PBSID) method was used for determining the linear dynamic model. The method provides a stable, timevarying, and individualized state-space model for predicting CGM measurements by taking the insulin infusion rates as the manipulated input variables. The CGM measures were used to determine the appropriate values for the PIC bounds and the risk indexes. The results indicated that the proposed method may control BG levels within the safe range without meal announcement.

Chakrabarty et al. [14] presented an event-triggered communication (ETC) algorithm for selective transmission of CGM values to the controller to reduce communication between the sensor and the controller. The CGM value is not transmitted to the controller if the current and predicted BG levels are in the safe zone. An observer-based MPC algorithm with event-triggered CGM communication to regulate the BG levels was deployed. The proposed method effectively reduced the sensor-controller transmissions in the hypoglycemic range and required less time than the standard MPC.

\section{B. Bayesian Optimization Approach}

Shi et al. [4] proposed the controller adaptation method based on the multivariate Bayesian optimization (BO) approach and the dynamic parameter selection module for solving the parameter adaptation problem. The dynamic parameter selection module determines the parameter that should be adjusted based on the performed diagnostics of the BG levels. The BO-based optimization module, shown in Fig. 2, automatically adjusts the selected parameter and optimizes an unknown cost function that determines the amount of glycemic regulation.

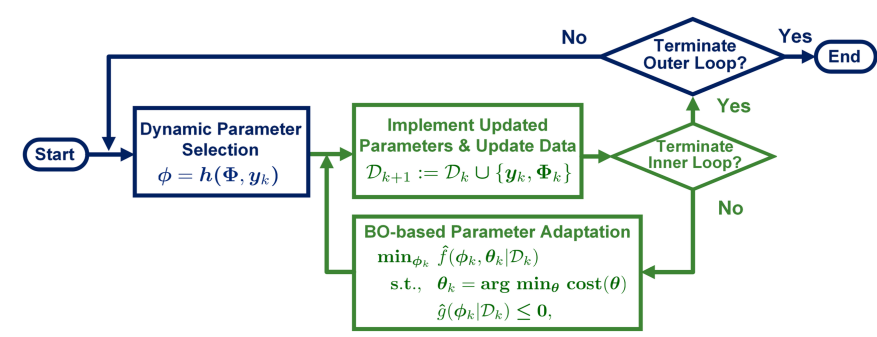

Fig. 2. The BO-based multivariate controller adaptation scheme. The dynamic parameter selection module (blue) and the optimization module (green) [4]. 
The two scenarios help verify the efficiency and robustness of the proposed algorithm. In the first scenario, the algorithm improves the rate of insulin delivery and reduces BG levels to the euglycemic range. In the second scenario, the algorithm improves the duration of the insulin delivery. Hence, the proposed method may properly adjust the parameters and their identifications in order to achieve regulation of adequate BG levels with no risk of hypoglycemia.

\section{Sliding Mode Control Approach}

Leyva et al. [15] presented methods for insulin stabilization based on positive sliding mode control (SMC) and the Lyapunov function (CLF) control theory. The cascade structure of the physiological model was used to improve the rate of stabilization of BG levels while the compartmental mathematical model was used to reproduce glucose metabolism and the insulin and glucagon dynamics. The proposed methods solve BG stabilization, maintain BG levels in a healthy physiological range, and avoid hypoglycemia. The CLF exhibited better results, improved convergence rate, and generated a continuous signal that prevented insulin accumulation.

To improve the regulation of the $\mathrm{BG}$ levels caused by unannounced physical activity, Beneyto et al. [16] enhanced the insulin-only controller using fast-acting $\mathrm{CHO}$ for the recommender system as shown in Fig. 3. The insulin-only controller is composed of two loops: a proportional-derivative (PD) controller with insulin feedback (IFB) and a safety auxiliary feedback element (SAFE) layer. The SAFE layer consists of insulin on board (IOB) constraints, sliding mode reference conditioning (SMRC) block, and a low-pass firstorder filter. The $\mathrm{CHO}$ controller is based on a predictive quantified proportional-derivative controller. The comparison between the original insulin-only controller and the combined insulin $\mathrm{CHO}$ recommender system showed that the novel combined system is able to reduce daily hypoglycemia episodes and increase the rate of insulin delivery per unit time within acceptable limits.

Moscardo et al. [17] extended the coordinated configuration (CC) control structure with insulin-on-board (IOB) limitation by using Sliding Mode Reference Conditioning (CC-SMRC) for coordinated control of glucose as shown in Fig. 4. Comparison between the CC and CC-SMRC control structures based on meals, snacks, and exercise scenarios indicates that the proposed CC control method with the IOB limitation has a slightly lower periods of hypoglycemia in the meal and meals plus snack scenarios. However, in the exercise scenario, the reduction was statistically significant. The rate of insulin delivery was better using the CC-SMRC control method during the exercise scenario than during the meals scenarios. During the most demanding exercise scenario, the insulin delivery levels were low and insufficient to prevent hypoglycemia.

\section{Proportional Integral Derivative Control Approach}

To reduce delay of the insulin absorption, Barnes and Jones [18] relied on a continuous intra-peritoneum insulin infusion (CIPII) method based on proportional integral derivative

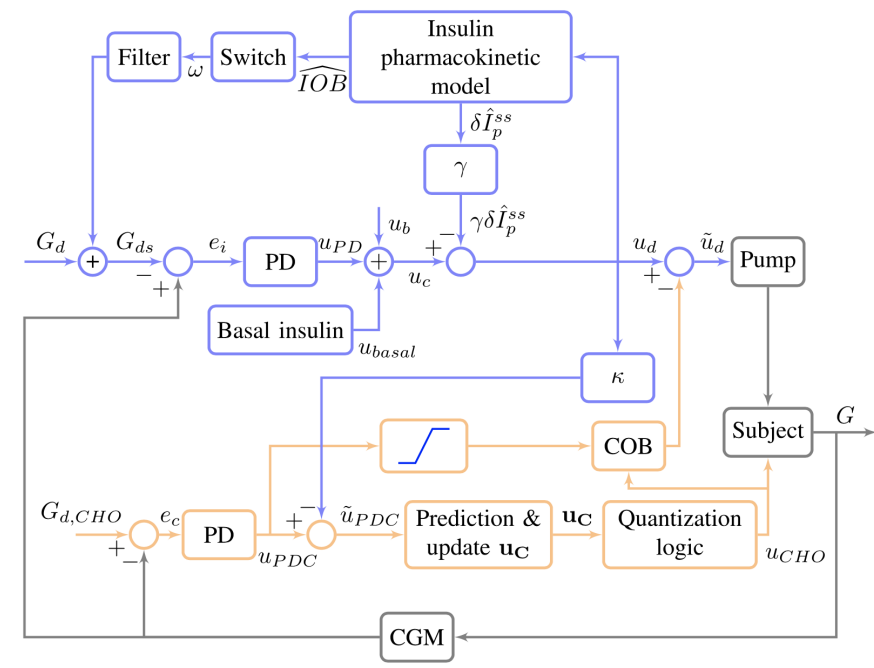

Fig. 3. The insulin-only controller (blue) with the $\mathrm{CHO}$ controller (orange) [16].

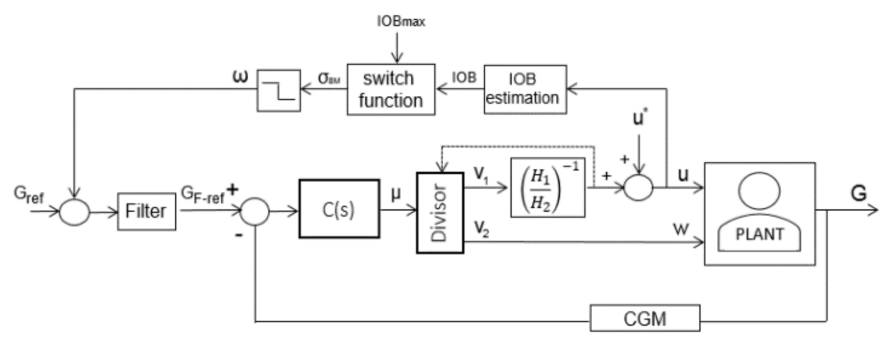

Fig. 4. The closed-loop system based on CC-SMRC controller [17].

(PID) controller. They introduced IMC-PID controller based on the Internal Model Control (IMC) tuning method that employs an inverter to realize the PID controller feedback. Time delay was modeled using the first-order with time delay (FOPTD) model with Pade approximation. Comparison between the subcutaneous insulin infusion (CSII) method based on the MPC controller and the continuous CIPII method indicated that the PID controller with the insulin feedback method may successfully control the BG oscillations.

Kushner et al. [19] presented novel non-deterministic datadriven model with a PID-based closed-loop system for BG levels control. Patient data, together with insulin and glucose data, were used in order to predict how the patients react under the PID-based closed-loop system. The results have shown that simple non-deterministic models may efficiently adjust key controller parameters and improve BG levels control.

\section{E. Active Disturbance Rejection Control Approach}

Cai et al. [20] proposed a method based on the active disturbance rejection control (ADRC) for regulation of $\mathrm{BG}$ levels by adding the insulin on board (IOB) and insulin delivery constraints to ensure the safety of the control algorithm. The proposed ADRC controller is composed of two modules: the ADRC module (composed of tracking differentiator (TD), 
extended state observer (ESO), and nonlinear feedback) and the constraints module (composed of the IOB, non-negative, and maximum input constraints). The controller achieved satisfactory performance for glucose regulation and rate of insulin delivery with no risk of hypoglycemia.

\section{F. Bioinspired AP Approach}

Güemes et al. [21] proposed an extension of the existing bihormonal bioinspired AP (BiAP) controller by adding a novel hybrid hormonal-insulin sensitivity glucose (InSiG) controller to determine insulin and glucagon doses and the desired insulin sensitivity (SI) from CGM measurements. The coordinated bihormonal BiAP controller determines insulin and glucagon doses while a standard proportional-derivative (SPD) controller determines the desired SI. A comparison between the InSiG controller and the coordinated bi-hormonal BiAP controller indicated that the InSiG controller achieves improved glucose control and maintains glucose levels within target with no risk of hypoglycemia. Using the $\mathrm{InSiG}$ controller reduced the total delivered insulin dose while the glucagon dose was significantly reduced. The disadvantage of the method is the unknown relationship between the magnitude of stimulation of the nervous system and the dynamics of insulin sensitivity.

\section{ApProAch BASED ON SENSITIVITy AnAlysis}

Staal et al. [22] investigated methods to improve recognition and estimation of the most appropriate model parameters used in a T1D mellitus simulator and thus reduce parameters of the critical models. They also investigated identifiability of nonlinear state-space model parameters. The nonlinear observability rank condition (NORC) was used for structural while sensitivity analysis and Fisher information matrix (FIM) were used for practical identifiabilities. A simplified model, derived from free-living data (CGM, SMBG, meal, or insulin data), proved useful for AP applications.

\section{Approaches Based on Filters}

In this Section, we present recently proposed methods based on Kalman and kernel filtering (KRLS) algorithms for glucose measurements, regulating insulin infusion rate, and online glucose prediction.

Ortmann et al. [1] improved the existing MPC controller using a new kernel function for the Gaussian process in order to address the measurement noise in case of unannounced meals. The Unscented Kalman Filter provides the state estimate used to extract data in order to change insulin sensitivity. Extracted data are then processed using Gaussian filter to predict future effects. The MPC optimizes the received data in order to calculate the volume of insulin injections. The collected training data become insensitive to noise after applying the Gaussian process. This causes the controller to be insensitive to unannounced meals. The proposed method, illustrated in Fig. 5, enables effective improvement of glucose control strategy and personalized health care.

For regulating basal insulin infusion rates, Fushimi et al. [23] integrated an automatic switching signal generator

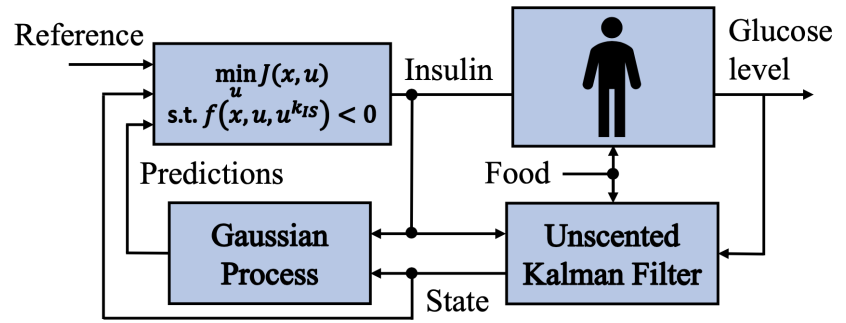

Fig. 5. The proposed method based on the Gaussian process, Unscented Kalman Filter, and MPC [1].

(SSG) into the Automatic Regulation of Glucose (ARG) algorithm and an advanced version of the switched Linear Quadratic Gaussian (SLQG) controller. The SSG module is based on the Kalman filter, which generates a filtered version of the BG levels. The proposed algorithm regulates the basal insulin infusion rate and generates insulin feedback during unannounced meals. Even though it introduces a large delay in selecting the controller mode after a meal, the proposed algorithm does not significantly increase hypoglycemia or hyperglycemia.

Fathi et al. [24] proposed a novel adaptive model-based algorithm for detecting unannounced meals by using the linear Kalman filter used to compute the evaluation of the glucose measurements. The statistical Generalized Likelihood Ratio Test (GLRT) was then employed (under the null hypothesis) to decide if the glucose measurements are affected by an unannounced meal. The threshold criterion was applied to GLRT in order to control and prevent the increase of BG levels. The results indicted that the proposed algorithm successfully detected unannounced meals without false positives.

Yu et al. [25] extended the adaptive kernel filtering (KRLS) algorithm with the sparsification criteria to improve computational efficiency in online glucose prediction. They combined the KRLS algorithm with the the approximate linear dependency (ALD) and the surprise criterion (SC) to design the online sparse ALD-KRLS and SC-KRLS algorithms. The proposed online adaptive method proved insensitive to abnormal or inaccurate CGM measurements and adaptable to prediction models. It effectively reduces the computational load and regulates the time delay in glucose nonlinear dynamics.

\section{Machine Learning Algorithms}

We review recently proposed machine learning methods [26] for detecting insulin pump malfunction, predicting the quality of overnight glycemic control, and solving the BG stability and gradient problems.

\section{A. Algorithms Based on Unsupervised Learning}

Meneghetti et al. [27] proposed an unsupervised modelfree approach to detect insulin pump malfunction by relying on data-driven techniques for anomaly detection (AD). The machine learning approaches were applied on the extracted set of features to detect anomalies using local outlier factor (LOF), connectivity-based outlier factor (COF), and isolation forest 
(iF/iForest). The proposed Four Time Series Data (4TSD) procedure was applied to LOF and COF to overcome correlations among time-closed samples. The optimal parameter configuration for LOF and iForest offered a satisfactory detection performance while maintaining high accuracy. When compared with the traditional multivariate control chart (MCC) method (used as a baseline approach), COF outperformed other methods while LOF and iForest offered a comparable performance. Furthermore, iForest was shown to be prone to errors and instabilities despite good performance.

\section{B. Algorithms Based on Binary Classification}

Güemes et al. [28] proposed a novel data-driven method in order to predict quality of overnight glycemic control by analyzing small dataset from the CGM measurements, meal intake, and insulin boluses. Machine learning algorithms for binary classification such as Random Forest Classifier (RFC), Artificial Neural Networks (ANN), Support Vector Machine (SVM), Linear Logistic Regression (LLR), and Extended Tree Classifier (ETC) were used to classify the quality of overnight glycaemic control. Previously processed data were used for the training and evaluation of binary classifiers. The proposed method is able to predict overnight BG levels within the target range. However, a larger dataset is needed in order to fully validate the proposed method. Thus, a more advanced classification algorithms such as LSTM networks may be more suitable for processing dataset sequences.

\section{Non-Linear Autoregressive Neural Network and Long Short-Term Memory}

Aliberti et al. [29] improved prediction accuracy and robustness of previous methods by applying Non-Linear Autoregressive (NAR) neural network and Long Short-Term Memory (LSTM) on BG signals, as illustrated in Fig. 6. NAR was used to solve BG stability issues while the LSTM addressed the exploding and disappearing gradient and to maintain longterm information over time.

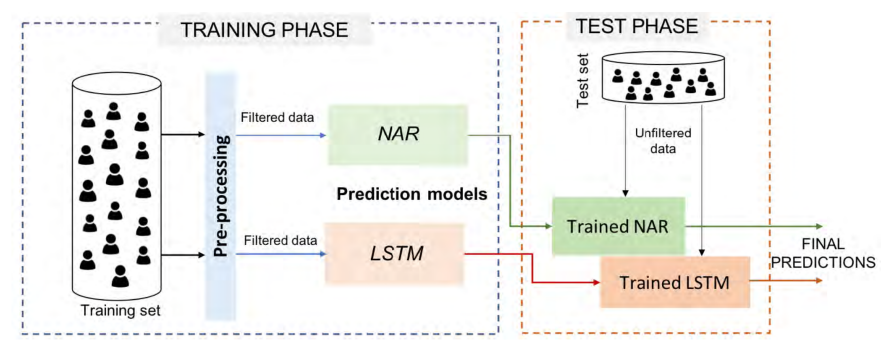

Fig. 6. Improved solution using NAR and LSTM methods [29].

Tikhonov regularization was applied as a pre-processing step for filtering signals using training data from CGM systems. To avoid over-fitting of parameters in the hardware implementation, the two-step design procedure was applied in case of the NAR network. In the first phase, an automated optimization strategy based on the Lipschitz method was applied to determine the delay of glucose signals to be used as regressors. Based on these results, three fully-connected NAR models were developed. In the second phase, an Optimal Brain Surgeon (OBS) method was implemented to minimize the error variation by using a recursive calculation of the inverse Hessian matrix applied to training data. The improved method eliminates redundant connections between the neurons without affecting prediction capability. Then, the three models were trained again with a Levenberg-Marquardt backpropagation procedure (LMBP) in order to obtain the normalized sum of squared errors (nSSE).

The co-dependence of the LSTM parameters was reduced by removing individual nodes and corresponding links at each training stage of the model. The Adam (adaptive moment estimation) method was then applied as an optimizer for adjusting individual learning rates for each parameter, which helped deal with non-stationary noise. When compared to the Recurrent Neural Networks (RNNs), LSTM proved resistant to the exploding and vanishing gradient problems.

The proposed methods were trained and tested on a large dataset of continuous signals from monitoring systems. They were compared with multi-patient techniques such as autoregressive (AR), feed-forward neural networks (FNNs), and recurrent neural networks (RNNs). The NAR model has shown good prediction accuracy only for a short-term period while the LSTM model exhibited very good performance for predicting both short-term and long-term BG levels.

Li et al. [30] proposed a convolutional recurrent neural network (CRNN) method for predicting BG levels using both in-silico and clinical datasets. The CRNN architecture consisted of a multi-layer convolutional neural network (CNN), a RNN layer with LSTM cells, and fully-connected layers. The CNN was employed to extracts features or patterns of the multi-dimensional time series while the modified RNN was used to analyze the previous sequential data and predict $\mathrm{BG}$ levels. The models for each diabetic subject were trained using individual patients' data. The root-mean-square error (RMSE) and mean absolute relative difference (MARD) indicators were used for the predicted and referenced glucose measurements and for evaluating the accuracy of the proposed algorithm. The proposed method predicted BG levels with high accuracy. The trained neural network was also developed for implementation on portable devices.

\section{Bioinspired Reinforcement Learning}

Lee et al. [31] proposed a novel AI-based bioinspired reinforcement learning (RL) approach for automated insulin infusion to maintain BG levels and robustness of the CGM sensor. The layer-wise relevance propagation (LRP) method was used to analyze input-output relevance and define the rate of insulin infusion. The LRP method based on AI-decisions provided information about insulin distribution, automated postprandial regulation, and sensor robustness. The proposed method was applied for training and evaluated on virtual patients. It was able to fully automate the control of BG levels in unannounced meals with no risks of hypoglycemia. 


\section{CONCLUSION}

In this paper, we reviewed recently proposed solutions for regulation and control of the insulin delivery in type 1 diabetes. The proposed methods are based on predictive control, sensitivity analysis, filters, and machine learning theory. The control approaches include model predictive control, Bayesian optimization, sliding mode control, proportional integral derivative control, active disturbance rejection control, and bioinspired artificial pancreas. Combination of common control methods have exhibited good results in controlling blood glucose levels. Other successful approaches include methods for identifiability analysis, methods based on filters, and learning algorithms. Novel deep and reinforcement learning algorithms promise improved performance due to the availability of larger experimental datasets and powerful hardware platforms.

\section{REFERENCES}

[1] L. Ortmann, D. Shi, E. Dassau, F. J. Doyle, B. J. E. Misgeld, and S. Leonhardt, "Automated insulin delivery for type 1 diabetes mellitus patients using Gaussian process-based model predictive control," in Proc. 2019 American Control Conf. (ACC), Philadelphia, PA, USA, Jul. 2019, pp. 4118-4123.

[2] F. Cairoli, G. Fenu, F. A. Pellegrino, and E. Salvato, "Model predictive control of glucose concentration based on signal temporal logic specifications," in Proc. 2019 6th Int. Conf. Control, Decision Inform. Technol. (CoDIT), Paris, France, Apr. 2019, pp. 714-719.

[3] R. A. DeFronzo, E. Ferrannini, P. Zimmet, and K. G. M. M. Alberti, International Textbook of Diabetes Mellitus, 4th ed. Oxford, UK: WileyBlackwell, 2015.

[4] D. Shi, E. Dassau, and F. J. Doyle, "A multivariate Bayesian optimization framework for long-term controller adaptation in artificial pancreas," in Proc. 2018 IEEE Conf. Decision Contr. (CDC), Miami Beach, FL, USA, Dec. 2018, pp. 276-283.

[5] P. Szcześniak, G. Tadra, and Z. Fedyczak, "Model predictive control of hybrid transformer with matrix converter," ACTA Polytechnica Hungarica, vol. 17, no. 1, pp. 25-40, 2020.

[6] D. Boiroux and J. B. Jørgensen, "Nonlinear model predictive control and artificial pancreas technologies," in Proc. 2018 IEEE Conf. Decision Control (CDC), Miami Beach, FL, USA, Dec. 2018, pp. 284-290.

[7] R. Hovorka, J. M. Allen, D. Elleri, L. J. Chassin, J. Harris, D. Xing, C. Kollman, T. Hovorka, A. M. F. Larsen, M. Nodale, A. D. Palma, M. E. Wilinska, C. L. Acerini, and D. B. Dunger, "Manual closed-loop insulin delivery in children and adolescents with type 1 diabetes: a phase 2 randomised crossover trial," The Lancet, vol. 375, no. 9716, pp. 743751, Feb. 2010.

[8] S. Schmidt, D. Boiroux, A. K. Duun-Henriksen, L. Frøssing, O. Skyggebjerg, J. B. Jørgensen, N. K. Poulsen, H. Madsen, S. Madsbad, and K. Nørgaard, "Model-based closed-loop glucose control in type 1 diabetes: the DiaCon experience," J. Diabetes Sci. Technol., vol. 7, no. 5, pp. 1255-1264, Sep. 2013.

[9] S. D. Favero, D. Bruttomesso, F. D. Palma, G. Lanzola, R. Visentin, A. Filippi, R. Scotton, C. Toffanin, M. Messori, S. Scarpellini, P. KeithHynes, B. P. Kovatchev, J. H. DeVries, E. Renard, L. Magni, A. Avogaro, and C. Cobelli, "First use of model predictive control in outpatient wearable artificial pancreas," Diabetes Care, vol. 37, no. 5, pp. 1212 1215, May 2014.

[10] S. Schmidt and K. Nørgaard, "Bolus calculators," J. Diabetes Sci. Technol., vol. 8, no. 5, pp. 1035-1041, May 2014.

[11] D. Shi, E. Dassau, and F. J. Doyle, "Adaptive zone model predictive control of artificial pancreas based on glucose- and velocity-dependent control penalties," IEEE Trans. Biomed. Eng., vol. 66, no. 4, pp. 10451054, Apr. 2019.

[12] A. Chakrabarty, D. Shi, S. Zavitsanou, F. J. Doyle, and E. Dassau, "Embedded model predictive control for a wearable artificial pancreas," IEEE Trans. Control Syst. Technol., pp. 1-8, Sep. 2019.
[13] M. Rashid, I. Hajizadeh, and A. Cinar, "Predictive control with variable delays in plasma insulin action for artificial pancreas," in Proc. 2018 IEEE Conf. Decision Control (CDC), Miami Beach, FL, USA, Dec. 2018, pp. 291-296.

[14] A. Chakrabarty, S. Zavitsanou, F. J. Doyle, and E. Dassau, "Model predictive control with event-triggered communication for an embedded artificial pancreas," in Proc. 2017 IEEE Conf. Control Technol. Appl. (CCTA), Mauna Lani, HI, USA, Aug. 2017, pp. 536-541.

[15] H. Leyva, G. Quiroz, F. A. Carrillo, and R. Femat, "Insulin stabilisation in artificial pancreas: a positive control approach," IET Control Theory Appl., vol. 13, no. 7, pp. 970-978, Apr. 2019.

[16] A. Beneyto, A. Bertachi, J. Bondia, and J. Vehi, "A new blood glucose control scheme for unannounced exercise in type 1 diabetic subjects," IEEE Trans. Control Syst. Technol., pp. 1-8, Nov. 2018.

[17] V. Moscardö, P. Herrero, J. L. Diez, M. Giménez, P. Rossetti, and J. Bondia, "In silico evaluation of a parallel control-based coordinated dual-hormone artificial pancreas with insulin on board limitation," in Proc. 2019 American Control Conf. (ACC), Philadelphia, PA, USA, Jul. 2019, pp. 4759-4764.

[18] A. J. Barnes and R. W. Jones, "PID-based glucose control using intraperitoneal insulin infusion: an in silico study," in Proc. 2019 14th IEEE Conf. Ind. Electron. Appl. (ICIEA), Xi'an, China, Jun. 2019, pp. 10571062.

[19] T. Kushner, D. Bortz, D. M. Maahs, and S. Sankaranarayanan, "A datadriven approach to artificial pancreas verification and synthesis." in Proc. 2018 ACM/IEEE 9th Int. Conf. Cyber-Physical Syst. (ICCPS), Porto, Portugal, Apr. 2018, pp. 242-252.

[20] D. Cai, J. Song, J. Wang, and D. Shi, "Glucose regulation for subjects with type 1 diabetes using active disturbance rejection control," in Proc. 2019 Chinese Control Conf. (CCC), Guangzhou, China, Jul. 2019, pp. 6970-6975.

[21] A. Güemes, P. Herrero, and P. Georgiou, "A novel glucose controller using insulin sensitivity modulation for management of type 1 diabetes," in Proc. 2019 IEEE Int. Symp. Circuits Syst. (ISCAS), Sapporo, Japan, May 2019.

[22] O. M. Staal, A. L. Fougner, S. Sælid, and Ø. Stavdahl, "Glucose-insulin metabolism model reduction and parameter selection using sensitivity analysis," in Proc. 2019 American Control Conf. (ACC), Philadelphia, PA, USA, Jul. 2019, pp. 4104-4111.

[23] E. Fushimi, P. Colmegna, H. D. Battista, F. Garelli, and R. SánchezPeña, "Unannounced meal analysis of the ARG algorithm," in Proc. 2019 American Control Conf. (ACC), Philadelphia, PA, USA, Jul. 2019, pp. 4740-4645.

[24] A. E. Fathi, E. Palisaitis, B. Boulet, L. Legault, and A. Haidar, "An unannounced meal detection module for artificial pancreas control systems," in Proc. 2019 American Control Conf. (ACC), Philadelphia, PA, USA, Jul. 2019, pp. 4130-4135.

[25] X. Yu, M. Rashid, J. Feng, N. Hobbs, I. Hajizadeh, S. Samadi, M. Sevil, C. Lazaro, Z. Maloney, E. Littlejohn, L. Quinn, and A. Cinar, "Online glucose prediction using computationally efficient sparse Kernel filtering algorithms in type-1 diabetes," IEEE Trans. Control Syst. Technol., pp. 1-13, Jun. 2018

[26] C. M. Bishop, Pattern Recognition and Machine Learning. Secaucus, NJ, USA: Springer-Verlag, 2006.

[27] L. Meneghetti, M. Terzi, S. D. Favero, and G. A. Susto, "Data-driven anomaly recognition for unsupervised model-free fault detection in artificial pancreas," IEEE Trans. Control Syst. Technol., pp. 1-15, Dec. 2018.

[28] A. Güemes, G. Cappon, B. Hernandez, M. Reddy, N. Oliver, P. Georgiou, and P. Herrero, "Predicting quality of overnight glycaemic control in type 1 diabetes using binary classifiers," IEEE J. Biomed. Health Inform., Sep. 2019.

[29] A. Aliberti, I. Pupillo, S. Terna, E. Macii, S. D. Cataldo, E. Patti, and A. Acquaviva, "A multi-patient data-driven approach to blood glucose prediction," IEEE Access, vol. 7, pp. 69311-69325, May 2019.

[30] K. Li, J. Daniels, C. Liu, P. Herrero, and P. Georgiou, "Convolutional recurrent neural networks for glucose prediction," IEEE J. Biomed. Health Inform., vol. 24, no. 2, pp. 603-613, Feb. 2020.

[31] S. Lee, J. Kim, S. W. Park, S. M. Jin, and S. M. Park, "Toward a fully automated artificial pancreas system using a bioinspired reinforcement learning design: in silico validation," IEEE J. Biomed. Health Inform. (Early Access), Jun. 2020. 